\title{
Investigation of Heschl's gyrus and planum temporale in patients with schizophrenia and bipolar disorder: A proton magnetic resonance spectroscopy study
}

\author{
M.İ. Atagün ${ }^{\text {a,b,* }}$, E.M. Şıkoğlu ${ }^{\text {c,d }}$, S.S. Can ${ }^{\text {a,b }}$, G. Karakaş-Uğurlu ${ }^{\text {a,b }}$, S. Ulusoy-Kaymak ${ }^{\text {, }}$, A. Çayköylü ${ }^{\text {a,b }}$, \\ O. Algin f,g , M.L. Phillips h , C.M. Moore ${ }^{\mathrm{c}, \mathrm{d}, \mathrm{e}}$, D. Öngür ${ }^{\mathrm{i}, \mathrm{j}}$
}

a Ylldırtm Beyazıt University, Faculty of Medicine, Department of Psychiatry, Ankara, Turkey

b Ankara Atatürk Training and Education Hospital, Psychiatry Clinic, Ankara, Turkey

c Center for Comparative NeuroImaging, University of Massachusetts Medical School, Worcester, MA, USA

d Department of Psychiatry, University of Massachusetts Medical School, Worcester, MA, USA

e Department of Radiology, University of Massachusetts Medical School, Worcester, MA, USA

${ }^{\mathrm{f}}$ Ankara Atatürk Training and Education Hospital, Radiology, Ankara, Turkey

${ }^{g}$ Bilkent University, National MR Research Center, Bilkent, Ankara, Turkey

${ }^{\text {h }}$ University of Pittsburgh, Medical School, Department of Psychiatry, Pittsburgh, PA, USA

${ }^{i}$ Harvard Medical School, Department of Psychiatry, Boston, MA, USA

${ }^{\mathrm{j}}$ McLean Hospital, Psychotic Disorders Division, Belmont, MA, USA

\section{A R T I C L E I N F O}

\section{Article history:}

Received 13 June 2014

Received in revised form 3 November 2014

Accepted 5 November 2014

Available online 3 December 2014

\section{Keywords:}

Schizophrenia

Bipolar disorder

Glutamate

Glutamine

Magnetic resonance spectroscopy

Superior temporal cortex

\begin{abstract}
A B S T R A C T
Background: Superior temporal cortices include brain regions dedicated to auditory processing and several lines of evidence suggest structural and functional abnormalities in both schizophrenia and bipolar disorder within this brain region. However, possible glutamatergic dysfunction within this region has not been investigated in adult patients.

Methods: Thirty patients with schizophrenia ( $38.67 \pm 12.46$ years of age), 28 euthymic patients with bipolar I disorder (35.32 \pm 9.12 years of age), and 30 age-, gender- and education-matched healthy controls were enrolled. Proton magnetic resonance spectroscopy data were acquired using a 3.0 T Siemens MAGNETOM TIM Trio MR system and single voxel Point REsolved Spectroscopy Sequence (PRESS) in order to quantify brain metabolites within the left and right Heschl's gyrus and planum temporale of superior temporal cortices.

Results: There were significant abnormalities in glutamate $(\mathrm{Glu})(\mathrm{F}(2,78)=8.52, \mathrm{p}<0.0001)$, N-acetyl aspartate $(\mathrm{tNAA})(\mathrm{F}(2,81)=5.73, \mathrm{p}=0.005)$, creatine $(\mathrm{tCr})(\mathrm{F}(2,83)=5.91, \mathrm{p}=0.004)$ and inositol $($ Ins $)(\mathrm{F}(2,82)=8.49$, $\mathrm{p}<0.0001$ ) concentrations in the left superior temporal cortex. In general, metabolite levels were lower for bipolar disorder patients when compared to healthy participants. Moreover, patients with bipolar disorder exhibited significantly lower $\mathrm{tCr}$ and Ins concentrations when compared to schizophrenia patients. In addition, we have found significant correlations between the superior temporal cortex metabolites and clinical measures.

Conclusion: As the left auditory cortices are associated with language and speech, left hemisphere specific abnormalities may have clinical significance. Our findings are suggestive of shared glutamatergic abnormalities in schizophrenia and bipolar disorder.
\end{abstract}

(c) 2014 Elsevier B.V. All rights reserved.

\section{Introduction}

Emil Kraepelin's dichotomous approach to discriminate schizophrenia and bipolar disorder relies on their different clinical courses. However, there has been discrepancy between Kraepelin's approach and studies showing co-aggregation of schizophrenia and bipolar disorder

\footnotetext{
* Corresponding author at: Cankiri Cd. Cicek Sk. No: 3, Altindag, Ulus, Ankara, Turkey. Tel.: +90 31232415 55/3016 1148; fax: +903123241518.

E-mail address: miatagun@ybu.edu.tr (M.İ. Atagün).
}

in families as well as shared susceptibility genes (Bramon and Sham, 2001; Craddock and Owen, 2005). The challenge arises due to the presence of overlapping symptoms between schizophrenia and bipolar disorder, but yet the desire for clear diagnostic categorization. Dimensional concepts suggest focusing on symptom domains rather than diagnostic categories (Cuthbert, 2014; Keshavan and Ongur, 2014). These symptom domains in conjunction with biological findings may provide insight for shared and distinct mechanisms underlying the disorders.

Superior temporal cortices host primary, secondary and association auditory cortices and have been implicated in the pathophysiology of 
schizophrenia. Both postmortem and in vivo measurements have shown reductions in volume, thickness and gray matter content of the superior temporal cortex in schizophrenia (Vita et al., 2012; Modinos et al., 2013). Longitudinal studies report progressive gray matter loss in the superior temporal gyrus and more precisely the Heschl's gyrus and planum temporale with progression to psychosis and development of delusions (Vita et al., 2012). Moreover, left superior temporal cortices have been associated with symptom domains such as auditory hallucinations (Dierks et al., 1999; Jardri et al., 2011; Kuhn and Gallinat, 2012; Modinos et al., 2013; Shinn et al., 2013) and thought disorder (Seese et al., 2011; Shenton et al., 1992) in psychosis. These findings suggest the superior temporal gyrus as a highly relevant location for the neurobiology and development of psychosis (Shenton et al., 1992; Rajarethinam et al., 2000; Takahashi et al., 2006; Seese et al., 2011). On the other hand, two meta-analyses of volumetric studies of superior temporal cortices did not report any significant differences between patients with bipolar disorder and healthy participants (Kempton et al., 2008; Arnone et al., 2009). However, primary and secondary auditory cortices are located in the region and functional studies consistently reported auditory processing disturbances in both schizophrenia (Dierks et al., 1999; Umbricht and Krljes, 2005; Domjan et al., 2012) and bipolar disorder (Hall et al., 2009; Oribe et al., 2010).

Since glutamate is the major excitatory neurotransmitter and since the EEG signal consists of excitatory end synaptic potentials, auditory processing deficits detected in both schizophrenia (Umbricht and Krljes, 2005; Oribe et al., 2010) and bipolar disorder (Hall et al., 2009; Ethridge et al., 2012; Atagun et al., 2014) could potentially be due to glutamatergic dysfunction in the auditory cortices (Javitt, 2009). Glutamate-modulating agents have been found to be efficacious in the treatment of mood disorders both in pre-clinical (Skolnick et al., 2009) and clinical studies (Sanacora et al., 2008; Machado-Vieira et al., 2012). Current psychotomimetics also modulate different components of the glutamatergic system (for reviews: (Machado-Vieira et al., 2012; Sanacora et al., 2008)). Chronic treatment with lamotrigine, valproate or lithium is likely to effect glutamatergic system through a variety of mechanisms (for reviews: (Colla et al., 2009; Gigante et al., 2012; Schifitto et al., 2009; Soeiro-de-Souza et al., 2013; Yatham et al., 2009)). Therefore, the nature and extent of the glutamatergic system abnormalities in patients with schizophrenia and mood disorders require further clarification.

Proton magnetic resonance spectroscopy ( ${ }^{1} \mathrm{H}$ MRS) is a non-invasive neuroimaging technique that can quantify in vivo neurochemical metabolites, including those related to the glutamatergic system. Glutamatergic neurotransmission is thought to be disturbed in both schizophrenia (Goff and Coyle, 2001; Paz et al., 2008; Javitt, 2009, 2010) and bipolar disorder (Sanacora et al., 2008; Machado-Vieira et al., 2012). Moreover, altered glutamatergic metabolites have been reported both in schizophrenia (Brugger et al., 2011; Marsman et al., 2013; Poels et al., 2014a,b) and bipolar disorder (Yildiz-Yesiloglu and Ankerst, 2006; Moore et al., 2007; Yuksel and Ongur, 2010; Ongur et al., 2011). Frontal, anterior cingulate and hippocampal regions are the most frequently studied brain regions and the findings were consistent across these brain regions. Only one study has investigated brain metabolites within the superior temporal gyrus (Seese et al., 2011) and reported a correlation between $\mathrm{N}$-acetyl aspartate (NAA) concentrations and thought disorder in children with schizophrenia. However, to our knowledge, no single ${ }^{1} \mathrm{H}$ MRS study has investigated the superior temporal region and compared the findings in adult patients with schizophrenia and patients with bipolar disorder.

Hence, in this study, we utilized ${ }^{1} \mathrm{H}$ MRS to examine neurometabolic changes within superior temporal cortices of adults with schizophrenia or bipolar disorder. Studies by different research modalities consistently report structural and functional abnormalities of auditory cortices, thus we expected to see abnormalities within the neurochemical profile of this region in schizophrenia and/or bipolar disorder.

\section{Methods}

\subsection{Participants and study procedures}

Thirty-five stable patients with paranoid schizophrenia (male or female; between ages of 18-59 years old) and 37 euthymic patients with bipolar disorder (male or female; between ages of 20-54 years old) were recruited for the study from the outpatient unit of Ankara Ataturk Training and Education Hospital, Ankara, Turkey. Five of the schizophrenia and 3 of the bipolar patients could not attend the MRI session and hence were excluded. Moreover, 5 of the bipolar patients had bipolar disorder II and therefore were not included in the analysis and we could not acquire neuroimaging data from 1 of the bipolar patients due to technical issues. In addition, 30 age-, gender-, and educationmatched healthy participants (male or female; between ages of 18-58 years old) were enrolled in the study. All participants provided written consent upon receiving detailed information about the study. The Ethical Committee of Yıldırım Beyazıt University approved the study.

All participants underwent a clinical interview administered by a psychiatrist (MIA) and diagnoses were checked with the Structured Clinical Interview according to the DSM-IV (SCID-I) (First et al., 1996). The clinical interview assessed the participants' demographics and medical/psychiatric history. Patients with bipolar disorder were evaluated using the Young Mania Rating Scale (YMRS) (Young et al., 1978) and the Hamilton Depression Rating Scale (HDRS) (Hamilton, 1960). Schizophrenia patients were evaluated with Scale for the Assessment of Positive Symptoms (SAPS) (including subscores for delusion and hallucination) (Andreasen, 1984) and Scale for the Assessment of Negative Symptoms (SANS) (Andreasen, 1983). All patients were also evaluated with the Brief Psychiatric Rating Scale (BPRS) (Overall and Gorham, 1962) and the Edinburgh Handedness Inventory (EHI) (Oldfield, 1971). All participants were free of any brain damage/surgeries or metabolic systemic diseases such as diabetes mellitus or hypertension or current or lifetime psychiatric comorbidity or substance abuse. Psychotropic medications except for benzodiazepines were allowed. Exclusion criteria also included any contraindications to MRI scan. In addition deafness or heading aids were excluded to avoid any possible plasticity effects as a result of hearing disabilities, as we are particularly interested in the temporal lobe. Finally, all participants completed an MRI scanning session.

\subsection{Neurolmaging data acquisition}

Data were acquired on a 3.0 T Siemens MAGNETOM TIM Trio wholebody MR system (Siemens, Erlangen, Germany) with a thirty twochannel phased-array head coil at the UMRAM National Magnetic Resonance Research Center, Ankara, Turkey.

T1-weighted anatomical MRI (MPRAGE sequence, $256 \times 256$ voxels, TR: 2000 ms, TE: $3.02 \mathrm{~ms}$, FOV read: 215, FOV phase: 100, slice thickness: $0.84,192$ slices) were collected for diagnostic and localization purposes. Voxels $(20 \mathrm{~mm} \times 20 \mathrm{~mm} \times 20 \mathrm{~mm}$ ) were placed consecutively in the left and right Heschl's gyrus and planum temporale regions (Fig. 1) and proton magnetic resonance spectroscopy ( ${ }^{1} \mathrm{H}$ MRS) data were acquired using the single voxel Point REsolved Spectroscopy Sequence (PRESS) (TE $=30 \mathrm{~ms}, \mathrm{TR}=2000 \mathrm{~ms}, 128$ averages) in order to quantify brain metabolites (Fig. 1 ). Manually adjusted $\mathrm{B}_{0}$ shimming was applied around the voxel as implemented on our MR system.

\subsection{NeuroImaging data analysis}

The proton spectra were fit using LCModel (Version 6.3.0) to quantify the metabolite concentrations. LCModel analyzes in vivo proton spectra as a linear combination of model in vitro spectra from individual metabolite solutions (Provencher, 1993, 2001) by utilizing built-in (simulated) radial basis sets. The basis set used for this study included alanine, aspartate, creatine $(\mathrm{Cr})$, phosphocreatine $(\mathrm{PCr}), \gamma$-aminobutyric acid (GABA), 


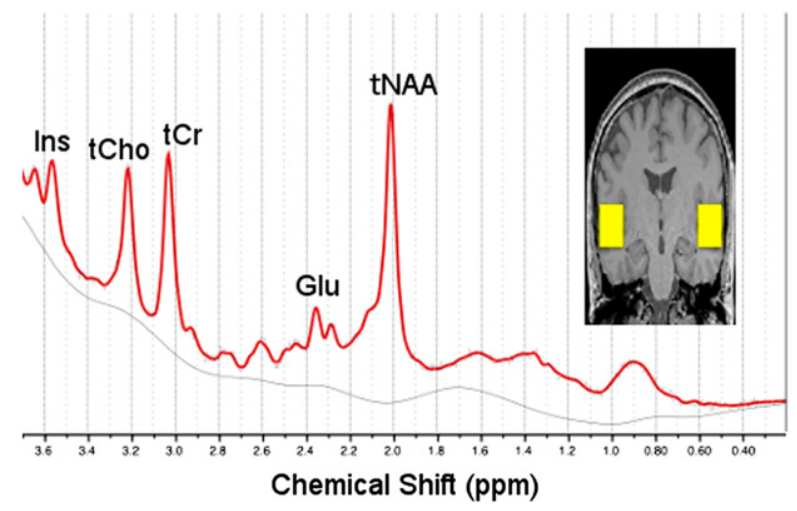

Fig. 1. Representative proton spectrum and LCModel fit (red) with sample placement of voxels (yellow) in the left and right superior temporal gyri.

glucose, glutamate (Glu), glutamine (Gln), glycerophosphocholine (GPC), phosphocholine (PCh), glutathione (GSH), inositol (Ins), lactate, $\mathrm{N}$-acetyl aspartate (NAA), N-acetyl aspartyl glutamate (NAAG), scylloinositol, taurine as well as macromolecules and lipids. Total NAA (tNAA, NAA and NAAG), Glu, Glx (Glu and Gln), total creatine (tCr, $\mathrm{Cr}$ and $\mathrm{PCr}$ ), total choline (tCh, GPC and PCh), and Ins were quantified and fits with Cramer-Rao lower bounds (CRLB, estimated error of the metabolite quantification) of greater than $10 \%$ were classified as not reliably detected and excluded from further analysis.

The structural T1-weighted images were segmented using SPM8 (Statistical Parameter Mapping - Welcome Department of Imaging Neuroscience, London, UK; (http://www.fil.ion.ucl.ac.uk/spm/software/spm8/)) to determine the gray matter, white matter and CSF contributions to the voxel of interest.

\subsection{Statistics}

Statistical analysis was performed using SPSS 21 (Armonk, NY: IBM Corp.). First, we conducted an outlier analysis for each metabolite within each group of participants. The values that were two standard deviations away from the mean of their corresponding groups were eliminated from further analysis. The remaining data were subjected to univariate ANOVA to compare the findings across 3 groups, i.e. schizophrenia patients vs. patients with bipolar disorder vs. healthy participants; and metabolite levels were the dependent factors for each comparison. Since we have 6 different metabolites (i.e. tNAA, Glu, Glx, tCr, tCho and Ins), to account for the multiple comparisons we considered $\mathrm{p}<0.008$ to be significant. We also performed post-hoc analyses with Bonferonni corrections and for this part of the analysis we considered $\mathrm{p}<0.05$ to be significant. In addition, we conducted an ANCOVA to investigate the potential covariates that have clinical importance and cover a range of values within each group (i.e. age of onset and duration of disorder as well as medication effects). Further, we conducted a Pearson's correlation analysis to explore the relationship between the brain metabolites and the clinical measures and for these analyses we considered $\mathrm{p}<0.05$ to be significant.

\section{Results}

\subsection{Participant demographics}

Thirty remitted patients with paranoid schizophrenia (18 male; average age: $38.7 \pm 12.5$ years) and 28 euthymic patients with bipolar disorder (13 male; average age: $35.3 \pm 9.1$ years) and 30 healthy participants ( 13 male; average age: $32.8 \pm 10.7$ years) were included in the study. There were no significant differences in any of the demographic variables across the groups $(p>0.05)$. Age of onset varied between the ages of 10 and 38 years for schizophrenia patients (average age of onset: $22.5 \pm 6.4$ years), and ages of 12 and 48 years (average age of onset: $23.6 \pm 8.7$ years) for patients with bipolar disorder. Schizophrenia patients scored significantly higher than patients with bipolar disorder in BPRS $(F(1,56)=29.45, \mathrm{p}<0.0001)$. The demographic and clinical characteristics of the sample are listed in Table 1.

In the schizophrenia group, there were three medication-free patients, 26 patients were on various mono-therapy antipsychotic medications and only 1 patient was taking both antipsychotic and valproate together. In the bipolar disorder group, there were five medication-free patients; the rest were on medication (valproate mono-therapy $(\mathrm{n}=3)$, combination therapy of valproate and antipsychotics $(n=3)$, lithium monotherapy $(n=6)$, combination therapy of lithium and antipsychotics $(n=3)$, combination of valproate, lithium and antipsychotics $(\mathrm{n}=1)$, antipsychotic mono-therapy $(\mathrm{n}=7)$ ).

\subsection{Brain metabolites}

We evaluated the brain metabolites from the left and right Heschl's gyrus and planum temporale regions separately. Statistically significant findings are summarized in Table 2.

Within the left hemisphere voxel, Glu $(F(2,78)=8.52, \mathrm{p}<0.0001)$ (Fig. $2 \mathrm{~A})$, $\mathrm{tNAA}(\mathrm{F}(2,81)=5.73, \mathrm{p}=0.005)$ (Fig. $2 \mathrm{C}), \operatorname{tCr}(\mathrm{F}(2,83)=$ 5.91, $\mathrm{p}=0.004$ ) (Fig. 2E) and Ins $(\mathrm{F}(2,82)=8.49, \mathrm{p}<0.0001$ ) (Fig. 2G) levels were statistically significantly different among the three groups. Post-hoc analysis revealed that tNAA, tCr and Ins levels were similar between schizophrenia patients and healthy participants ( $p>0.1$ ), but Glu levels were lower for schizophrenia patients than that of healthy comparisons at a trend level $(p=0.1)$. For Glu, tNAA, $\mathrm{tCr}$ and Ins, patients with bipolar disorder exhibited lower levels when compared to healthy participants (Glu: $\mathrm{p}<0.0001$; tNAA: $\mathrm{p}=0.004$; $\mathrm{tCr}: \mathrm{p}=0.008$; Ins: $\mathrm{p}=0.003$ ). In addition, $\mathrm{tCr}$ and Ins levels were higher for patients with schizophrenia when compared to patients with bipolar disorder ( $\mathrm{tCr}: \mathrm{p}=0.016$; Ins: $\mathrm{p}=0.001$ ). Due to the wide ranges in age at onset, duration of illness, and medication status, we further investigated the differences in Glu, tNAA, tCr and Ins among patients with bipolar disorder and schizophrenia patients by accounting for age onset, duration of disorder and the chlorpromazine equivalent values of medicated patients; results are summarized in Table 2.

On the other hand, there were no statistically significant metabolite differences within the right hemisphere voxel (Glu: Fig. 2B; tNAA: Fig. 2D; tCr: Fig. 2F; Ins: Fig. 2H).

Since there was no statistically significant differences between groups in terms of voxel tissue content (left hemisphere: gray matter $-(\mathrm{F}(2,84)=0.73, \mathrm{p}=0.48)$, white matter $-(\mathrm{F}(2,84)=0.22, \mathrm{p}=$ $0.81)$, csf $-\mathrm{F}(2,84)=0.78, \mathrm{p}=0.46$; right hemisphere: gray matter $-(\mathrm{F}(2,84)=0.25, \mathrm{p}=0.78)$, white matter $-(\mathrm{F}(2,84)=0.75, \mathrm{p}=$ $0.47), \operatorname{csf}-\mathrm{F}(2,84)=1.98, \mathrm{p}=0.15)$ we did not factor gray matter/ white matter/csf amounts into our analysis.

\subsection{Association between brain metabolites and clinical measures}

For schizophrenia patients, we performed exploratory correlation analyses to investigate the relationship between the brain metabolites and SAPS (total scores and subscores for bizarre behavior, formal thought disorder, inappropriate affect, delusion, and hallucination) and SANS (total scores and subscores for avolition, affective flattening, anhedonia, alogia, and attention).

Glu showed relationships with SANS affective flattening $(r=0.44$, $\mathrm{p}=0.02)$ and attention $(\mathrm{r}=0.44, \mathrm{p}=0.02)$ subscores, only for the right hemisphere. tNAA exhibited inverse relationships with SANS alogia $(r=-0.44, p=0.02)$ and attention subscores $(r=-0.44$, $\mathrm{p}=0.02$ ) in the left hemisphere. $\mathrm{tCr}$ had inverse correlations with SAPS total score $(\mathrm{r}=-0.37, \mathrm{p}=0.05)$, SAPS delusion subscore $(\mathrm{r}=$ $-0.46, \mathrm{p}=0.01)$, SANS anhedonia $(\mathrm{r}=-0.46, \mathrm{p}=0.01)$, SANS alogia 
Table 1

Clinical characteristics of participants.

\begin{tabular}{|c|c|c|c|c|c|c|}
\hline & & Schizophrenia $(\mathrm{n}=30)$ & Bipolar disorder $(\mathrm{n}=28)$ & Healthy controls $(\mathrm{n}=30)$ & $\mathrm{F} / \mathrm{X}^{2} / \mathrm{t}$ & $\mathrm{p}$ \\
\hline \multicolumn{2}{|l|}{ Age } & $38.7 \pm 12.5$ & $35.3 \pm 9.1$ & $32.8 \pm 10.7$ & 2.22 & 0.115 \\
\hline \multicolumn{2}{|l|}{ Gender (F) } & 12 & 15 & 17 & 1.88 & 0.391 \\
\hline \multicolumn{2}{|l|}{ Education } & $9.30 \pm 3.2$ & $10.89 \pm 4.9$ & $10.20 \pm 3.4$ & 1.24 & 0.294 \\
\hline \multicolumn{2}{|l|}{ Age at onset of disorder (years) } & $22.5 \pm 6.4$ & $23.6 \pm 8.7$ & & 0.89 & 0.578 \\
\hline \multicolumn{2}{|c|}{ Duration of disorder (months) } & $147.5 \pm 132.1$ & $92.2 \pm 107.7$ & & 3.19 & 0.089 \\
\hline \multicolumn{2}{|c|}{ Number of previous hospitalizations } & $2.4 \pm 5.1$ & $1.4 \pm 1.8$ & & 2.12 & 0.328 \\
\hline \multirow[t]{3}{*}{ Number of previous episodes } & Total & & $7.8 \pm 5.4$ & & & \\
\hline & Manic & & $2.8 \pm 2.2$ & & & \\
\hline & Depressive & & $4.2 \pm 3.4$ & & & \\
\hline \multicolumn{2}{|c|}{ Chlorpromazine equivalent (mg) } & $259.90 \pm 228.54$ & $144.4 \pm 231.83$ & & 0.005 & 0.061 \\
\hline \multicolumn{2}{|c|}{ Serum lithium levels $(\mathrm{mEq} / \mathrm{l})(\mathrm{n}=9)$} & & $0.67 \pm 0.22$ & & & \\
\hline \multicolumn{2}{|c|}{ Serum valproate levels $(\mu \mathrm{g} / \mathrm{ml})(\mathrm{n}=10)$} & & $73.53 \pm 27.08$ & & & \\
\hline \multicolumn{2}{|c|}{ BPRS } & $7.67 \pm 4.29$ & $2.43 \pm 2.86$ & & 3.20 & $<0.001$ \\
\hline \multicolumn{2}{|l|}{ YMRS } & & $1.07 \pm 1.61$ & & & \\
\hline \multicolumn{2}{|l|}{ HDRS } & & $3.46 \pm 3.51$ & & & \\
\hline \multirow[t]{6}{*}{ SAPS } & Total & $11.0 \pm 6.66$ & & & & \\
\hline & Bizarre behavior & $1.16 \pm 1.52$ & & & & \\
\hline & Formal thought disorder & $0.47 \pm 1.01$ & & & & \\
\hline & Inappropriate affect & $1.23 \pm 0.73$ & & & & \\
\hline & Delusions & $5.10 \pm 3.79$ & & & & \\
\hline & Hallucination & $2.60 \pm 3.84$ & & & & \\
\hline \multirow[t]{6}{*}{ SANS } & Total & $14.13 \pm 8.56$ & & & & \\
\hline & Avolition & $2.77 \pm 2.83$ & & & & \\
\hline & Affective flattening & $4.13 \pm 2.49$ & & & & \\
\hline & Anhedonia & $2.87 \pm 1.83$ & & & & \\
\hline & Alogia & $2.03 \pm 2.27$ & & & & \\
\hline & Attention & $2.33 \pm 1.86$ & & & & \\
\hline
\end{tabular}

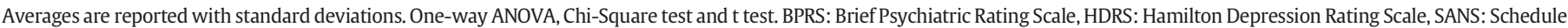
for the Assessment of Negative Symptoms, SAPS: Schedule for the Assessment of Positive Symptoms, YMRS: Young Mania Rating Scale.

$(\mathrm{r}=-0.37, \mathrm{p}=0.05)$ in the left hemisphere. Ins showed inverse relationships with SAPS delusion subscore $(\mathrm{r}=-0.42 \mathrm{p}=0.03)$, SANS anhedonia $(r=-0.47, p=0.01)$, and SANS alogia $(r=-0.39, p=0.04)$ in the left hemisphere.

For patients with bipolar disorder, we performed exploratory correlation analyses to investigate the relationship between the brain metabolites and YMRS, HDRS and BPRS. tNAA showed inverse relationships with HDRS for left $(\mathrm{r}=-0.46, \mathrm{p}=0.02)$ and right hemispheres $(\mathrm{r}=$ $-0.41, \mathrm{p}=0.03$ ). $\mathrm{tCr}$ also exhibited inverse correlation with HDRS for left $(r=-0.39, p=0.05)$ and right $(r=-0.44, p=0.02)$ hemispheres. In addition, Ins and HDRS had a reverse relationship in the right hemisphere $(\mathrm{r}=-0.52, \mathrm{p}=0.005)$.

\section{Discussion}

In this study, we observed decreased Glu concentrations within the left Heschl's gyrus and planum temporale of the superior temporal gyrus of patients with schizophrenia and bipolar disorder when compared to healthy participants. On the other hand, Glu, tNAA, tCr and Ins concentrations of the bipolar disorder group were lower when compared to schizophrenia patients and healthy participants. Moreover, $\mathrm{tCr}$ and Ins concentrations in the left superior temporal gyrus of schizophrenia patients were significantly higher than the concentrations measured from patients with bipolar disorder. All of these differences in metabolite levels between groups reached statistical significance only in the left superior temporal cortex, suggesting a possible location-

Table 2

${ }^{1} \mathrm{H}$ MRS findings.

\begin{tabular}{|c|c|c|c|c|c|c|}
\hline & & Schizophrenia $(\mathrm{n}=30)$ & Bipolar disorder $(\mathrm{n}=28)$ & Healthy controls $(\mathrm{n}=30)$ & $\mathrm{F}$ & $\mathrm{p}$ \\
\hline \multicolumn{7}{|l|}{ Left hemisphere } \\
\hline Glu & & $0.00088 \pm 0.00011$ & $0.00082 \pm 0.00010$ & $0.00095 \pm 0.00013$ & $\mathrm{~F}(2,78)=8.52$ & $<0.0001$ \\
\hline \multirow[t]{3}{*}{ Covariates: (related to disorder) } & Age onset & & & & $\mathrm{F}(1,50)=4.39$ & 0.041 \\
\hline & Duration & & & & $\mathrm{F}(1,50)=4.64$ & 0.036 \\
\hline & CPZE & & & & $\mathrm{F}(1,36)=5.78$ & 0.021 \\
\hline tNAA & & $0.00111 \pm 0.00016$ & $0.00104 \pm 0.00015$ & $0.00117 \pm 0.00013$ & $\mathrm{~F}(2,81)=5.73$ & 0.005 \\
\hline \multirow[t]{3}{*}{ Covariates: (related to disorder) } & Age onset & & & & $F(1,52)=3.53$ & 0.066 \\
\hline & Duration & & & & $\mathrm{F}(1,52)=3.73$ & 0.059 \\
\hline & CPZE & & & & $\mathrm{F}(1,36)=2.83$ & 0.101 \\
\hline $\mathrm{tCr}$ & & $0.00080 \pm 0.00011$ & $0.00071 \pm 0.00011$ & $0.00080 \pm 0.00011$ & $\mathrm{~F}(2,83)=5.91$ & 0.004 \\
\hline \multirow[t]{3}{*}{ Covariates: (related to disorder) } & Age onset & & & & $\mathrm{F}(1,52)=8.91$ & 0.004 \\
\hline & Duration & & & & $\mathrm{F}(1,52)=7.26$ & 0.009 \\
\hline & CPZE & & & & $\mathrm{F}(1,36)=6.40$ & 0.016 \\
\hline Ins & & $0.00067 \pm 0.00010$ & $0.00057 \pm 0.00012$ & $0.00067 \pm 0.00011$ & $\mathrm{~F}(2,82)=8.49$ & $<0.0001$ \\
\hline \multirow[t]{3}{*}{ Covariates: (related to disorder) } & Age onset & & & & $F(1,52)=14.48$ & $<0.0001$ \\
\hline & Duration & & & & $F(1,52)=11.25$ & 0.001 \\
\hline & CPZE & & & & $F(1,35)=6.64$ & 0.014 \\
\hline
\end{tabular}

Absolute quantity averages are reported with standard deviations. Univariate ANOVA. CPZE: chlorpromazine equivalent. 
A)

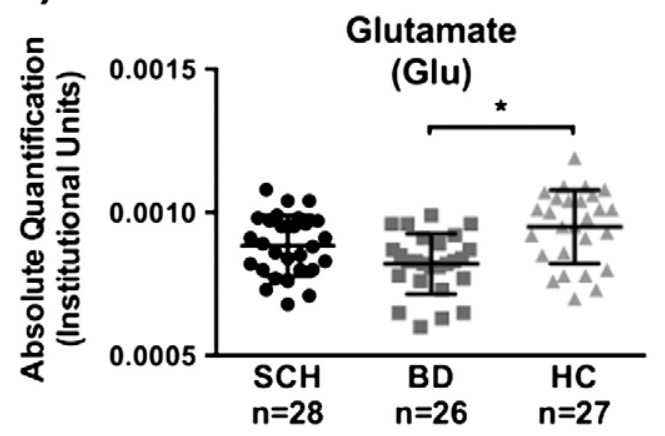

C)

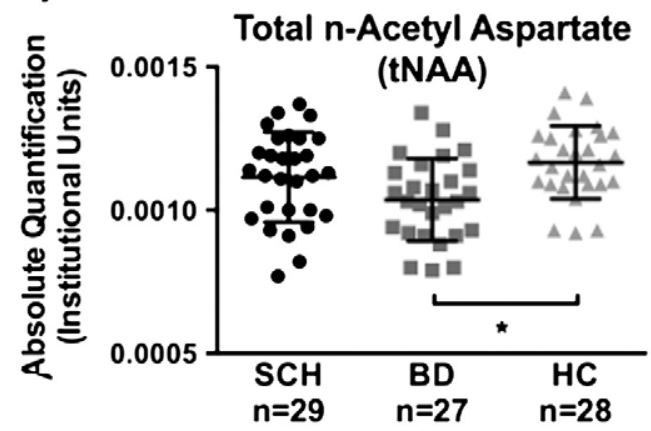

E)

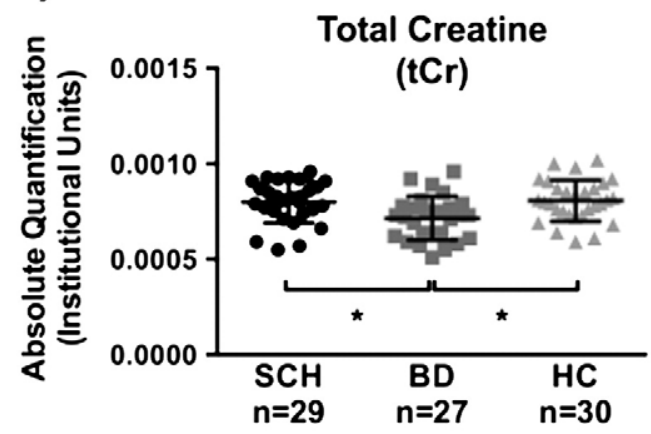

G)

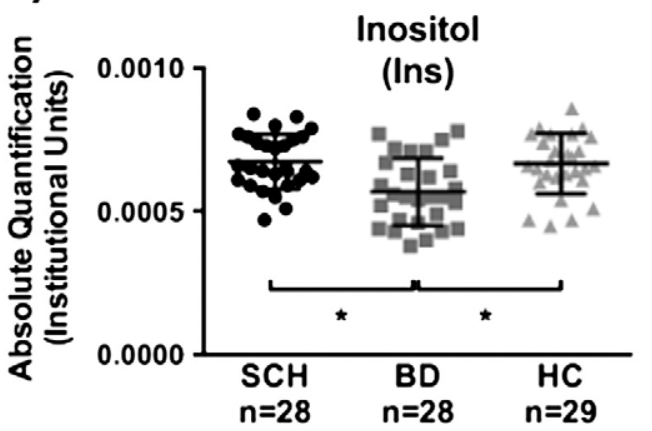

B)

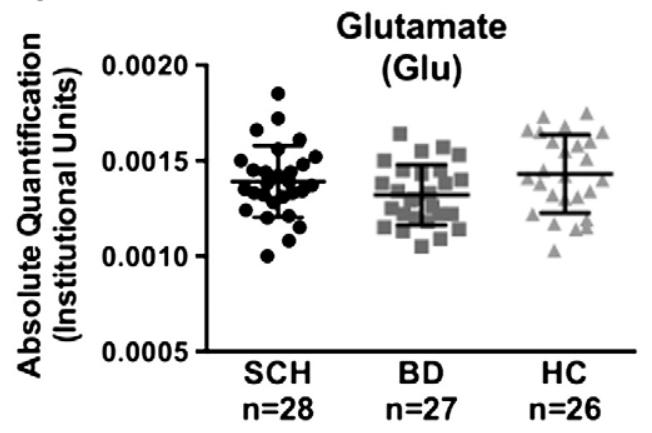

D)

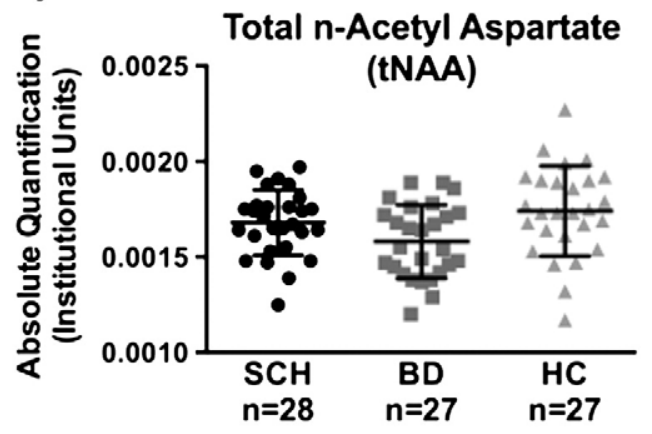

F)

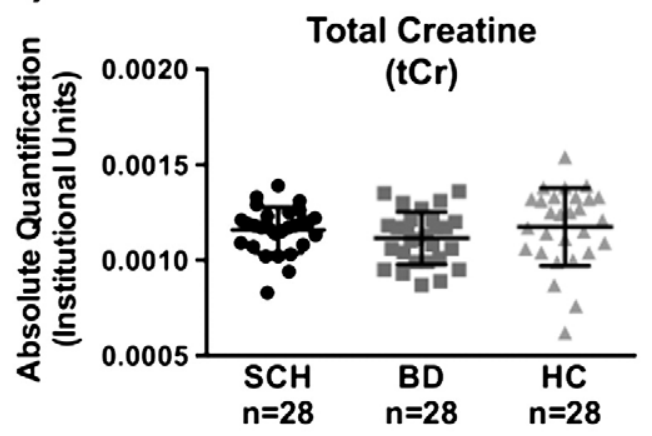

H)

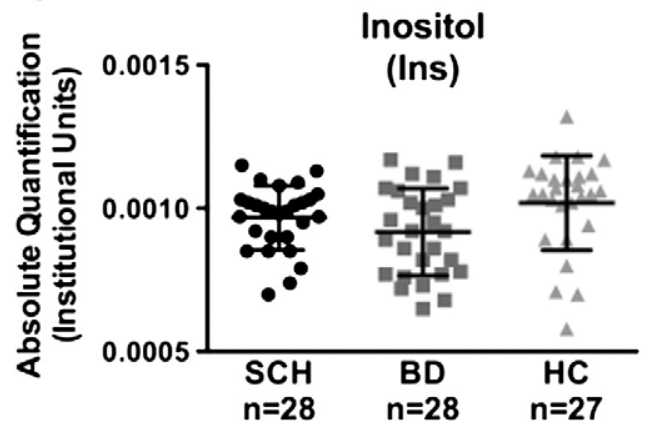

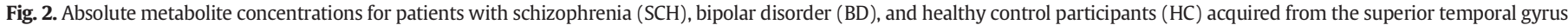

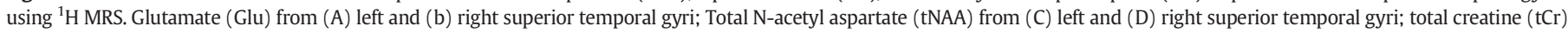

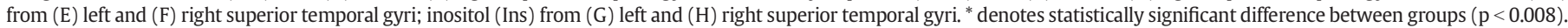

specific abnormality within the dominant hemisphere of auditory cortices for schizophrenia and bipolar disorder patients. It has been shown that auditory cortices are functionally lateralized (Zatorre et al., 1992; Kell et al., 2011) and the auditory cortex of the dominant hemisphere is associated with psychiatric disorders (Dierks et al., 1999; Plaze et al., 2006; Shinn et al., 2013).

Previous studies have reported glutamatergic abnormalities related to clinical progression in schizophrenia observed within different 
brain regions such as the anterior cingulate cortex (Goff and Coyle, 2001; Clinton and Meador-Woodruff, 2004; Kugaya and Sanacora, 2005; Javitt et al., 2012). Specifically, schizophrenia patients have exhibited increased concentrations of glutamatergic metabolites if the patients were in a first-episode and conversely, decreased concentrations if the patients had a chronic disease (Ohrmann et al., 2005; Ohrmann et al., 2008; Marsman et al., 2013; Schwerk et al., 2014). A recent study, investigating schizophrenia patients showed elevated Gln and Gln-to-Glu ratio but no significant difference in Glu levels in patients when compared to healthy comparisons (Bustillo et al., 2014). Our findings in a cohort of chronic schizophrenia patients exhibited decreased Glu concentrations at trend level for schizophrenia patients when compared to healthy participants and no change for Glx concentrations were observed.

Previous reports did not find any difference in Glu concentrations between patients with bipolar disorder and healthy participants (Gigante et al., 2012); however they consistently showed increase in Glx concentration for bipolar disorder patients, regardless of mood state (Yuksel and Ongur, 2010; Gigante et al., 2012). Although alterations in Glx have been suggested to mostly reflect changes in Glu levels (Dager et al., 2004), previous reports are suggestive of abnormalities in other components of the composite Glx peak, such as Gln (Moore et al., 2007), rather than Glu. However, our findings showed significant difference in Glu levels but not in Glx concentrations.

Glutamate turnover may be altered in different ways, resulting in changes in Gln-to-Glu ratio, depending on the disease state, i.e. manic (Ongur et al., 2008) or depressive (Auer et al., 2000) phases of bipolar disorder (reviewed in (Yuksel and Ongur, 2010)). These findings suggest an alteration in glutamatergic dysfunction between mania and depression for bipolar disorder. Glial cells are responsible for Glu-Gln cycling (Tansey et al., 1991; Cooper et al., 2003); therefore glial abnormalities are potentially the basis of differences between mania and depression in bipolar disorder (Ongur et al., 1998). Unfortunately, the field strength of the MRI scanner used in the current study did not allow us to reliably quantify Gln concentrations. We can only speculate that since the Glx levels did not differ between groups, but Glu concentrations were lower for both schizophrenia and bipolar disorder patients when compared to healthy participants, Gln levels may have been elevated. Taken together, it is possible to suggest that abnormalities in the Gln/ Glu cycle might be a cause of the observed reduction in Glu levels for the patients with bipolar disorder.

NAA is known to be lower in schizophrenia and bipolar disorder patients (Steen et al., 2005; Yildiz-Yesiloglu and Ankerst, 2006; Kraguljac et al., 2012a,b). Our investigation did not reveal any significant abnormality in tNAA concentrations in schizophrenia patients, possibly due to medication effects (Szulc et al., 2011; Kraguljac et al., 2012a; Szulc et al., 2013), but exhibited reduced tNAA levels for bipolar patients. $\mathrm{tCr}$ and Ins levels remain unchanged in schizophrenia (Schwerk et al., 2014) and our findings confirm this statement. However, there are a few studies that found reduced $\mathrm{tCr}$ levels in dorsolateral prefrontal cortex, anterior cingulate cortex, hippocampus and basal ganglia of schizophrenia patients (Ohrmann et al., 2005; Ohrmann et al., 2007; Ongur et al., 2009). In addition, the literature is not conclusive about the concentrations of $\mathrm{Cr}$ and Ins for patients with bipolar disorder (Yildiz-Yesiloglu and Ankerst, 2006; Frey et al., 2007; Theberge et al., 2007; Ongur et al., 2009; Silverstone and McGrath, 2009; Kraguljac et al., 2012a; Sikoglu et al., 2013). The decrease in tNAA, tCr and Ins that we observed in patients with bipolar disorder compared to healthy participants may be a region specific disturbance in this disorder, as we investigated an understudied brain area, i.e. the left superior temporal cortex. Regardless, these abnormalities are suggestive of abnormalities in neurochemicals other than glutamate in bipolar disorder.

The comparison of findings from patients with schizophrenia and bipolar disorder revealed significant differences only for $\mathrm{tCr}$ and Ins levels. The auditory system may show different abnormalities at multiple levels both in schizophrenia and bipolar disorder (Skold et al., 2014).
Our observations may imply that there exist common, i.e. glutamatergic dysfunction, as well as distinct abnormalities, i.e. in brain bioenergetics in schizophrenia and bipolar disorder within the auditory system.

Previous studies have focused primarily on higher order cortices, such as the frontal lobe where different channels of information converge and hence conduct serial and parallel processing together. However, lower order sensory areas, such as the auditory cortices have simpler receptive fields with only serial processing capabilities (Yamada and Wilber, 1990; Rauschecker and Scott, 2009; Homan et al., 2014). Given the fact that the pathophysiology of psychiatric disorders is so complicated and these disorders may have different influences on different neural systems and circuits, investigating the primary sensory areas may provide an advantage. Therefore, in this study we focused on the superior temporal gyrus to acquire ${ }^{1} \mathrm{H}$ MRS data.

Previous studies in the left STG in schizophrenia revealed correlations with clinical data, which suggests a relationship between the region and the effects of clinical factors. A ${ }^{31} \mathrm{P}$ MRS study reported correlation between the hallucination score of the Positive and Negative Syndrome Scale (PANSS) and phosphomonoesters and phosphodiesters (Nenadic et al., 2014). A recent ${ }^{1} \mathrm{H}$ MRS study comparing hallucinations in the STG reported that NAA/Cr ratio correlated with total and negative subscore of the PANSS in schizophrenia (Homan et al., 2014). Neuronal activation patterns for patients with auditory hallucinations were more prominent for the left hemisphere when compared to non-hallucinating patients as shown by functional neuroimaging studies with auditory tasks (Dierks et al., 1999; Shinn et al., 2013), which may indicate the relevance of the left auditory cortex. Besides, the auditory processing deficits in functional studies (Hall et al., 2009; Oribe et al., 2010; Domjan et al., 2012; Atagun et al., 2013; Atagun et al., 2014) point to abnormalities of the auditory cortices both in schizophrenia and bipolar disorder. In this study, both schizophrenia and bipolar disorder patients exhibited relationships between the studied metabolites and some of the clinical measures, especially on the left hemisphere. For example, HDRS scores correlated $\mathrm{tCr}$ and Ins concentrations in superior temporal cortices. This is particularly important as it may indicate a relationship between the metabolite levels and mood status.

\section{Limitations}

The current study had several limitations. First, our patients were patients with chronic disease and all were medicated. Although there was no statistically significant medication effect, small numbers of patients were taking each individual medication; therefore we cannot exclude medication effects conclusively. It is still possible that some of the metabolites were altered due to medications. Second, at $3 \mathrm{~T}$ magnetic field strength, some of the metabolite peaks in the ${ }^{1} \mathrm{H}-\mathrm{MRS}$ spectrum cannot be decomposed. In spite of the high quality of the glutamate output, it is difficult to completely discriminate glutamate from glutamine and GABA signal amplitudes, thus we could only identify Glu and Glx levels. Third, $8 \mathrm{~cm}^{3}$ voxel volumes cover an area larger than the auditory cortices alone.

\section{Conclusion}

Overlapping symptoms and intermediate diagnoses suggest that schizophrenia and bipolar disorder are disorders on a continuum. Genome wide association studies have identified shared risk genes in schizophrenia and bipolar disorder involving glutamatergic metabolism and cascades (see review; Coyle, 2006), which are in line with the glutamatergic abnormalities we have observed. Given that neurotransmission and intracellular functions are two major functions of glutamatergic metabolites, future studies focusing on glutamatergic abnormalities may provide novel targets for treatment development for both schizophrenia and bipolar disorder. In addition, this study reports a significant difference between creatine and inositol levels in 
patients with schizophrenia and bipolar disorder in the left superior temporal region. Taken together, there may be both shared and distinct mechanistic abnormalities within auditory cortices underlying pathophysiologies of schizophrenia and bipolar disorder. Therefore, our findings may suggest biological markers that may quantitatively distinguish the two disorders, and might be of importance for future studies.

\section{Role of funding source}

The study was funded by the Scientific Research Projects Committee of the Yıldırım Beyazıt University (project \#803) and NIMH grants to CMM (MH073998) and to DÖ (MH094594).

\section{Contributions}

MİA: Conceptualized and designed the study, prepared the initial protocol, conducted the psychiatric assessments of the participants, acquired the MRI data with support from MR technicians, helped with the statistical analysis, interpreted the data and wrote the manuscript.

EMS: Aided the design of the study, analyzed the MRS data, performed the statistical analysis, prepared figures and tables, helped with interpretation of the data and writing the manuscript.

SSC: Referred patients and helped Dr. Atagün to enroll participants.

GKU: Referred patients and helped Dr. Atagün to enroll participants.

SUK: Referred patients and helped Dr. Atagün to enroll participants.

AC: Supervised the study and the department which patients were enrolled.

OA: Helped the adjustments of the MRI protocol, checked the T1 and T2 images of the subjects.

MLP: Provided scientific supervision of the study, reviewed the initial protocol and the manuscript.

CMM: Provided scientific supervision for designing the study, analyzing and interpreting the data, critically reviewed the manuscript.

DÖ: Provided scientific supervision for conceptualizing and designing the study and the initial protocol, as well as interpreting the data, critically reviewed the manuscript.

\section{Conflict of interest}

The author DÖ participated in the Scientific Advisory Board for Lilly Inc. in 2013. Other authors do not report any conflict of interest.

\section{Acknowledgment}

MRI scans were conducted at the National Magnetic Resonance Research Center (UMRAM) of the Bilkent University (Ankara, Turkey) with support from the staff led by Prof. Dr. Ergin Atalar. In addition, we also appreciate technical help regarding voxel segmentation provided by Dinesh Deelchand, Dr. Uzay Emir and Dr. Gulin Oz from the Center for Magnetic Resonance Research, Minneapolis, MN, USA.

\section{References}

Andreasen, N.C., 1983. The Scale for Assessment of Negative Symptoms (SANS). University of Iowa, Iowa City, IA.

Andreasen, N.C., 1984. The Scale for the Assessment of Positive Symptoms (SAPS). University of Iowa, Iowa City, IA

Arnone, D., Cavanagh, J., Gerber, D., Lawrie, S.M., Ebmeier, K.P., McIntosh, A.M., 2009. Magnetic resonance imaging studies in bipolar disorder and schizophrenia: metaanalysis. Br. J. Psychiatry 195 (3), 194-201.

Atagun, M.I., Guntekin, B., Ozerdem, A., Tulay, E., Basar, E., 2013. Decrease of theta response in euthymic bipolar patients during an oddball paradigm. Cogn. Neurodyn. 7 (3), 213-223.

Atagun, M.I., Guntekin, B., Masali, B., Tulay, E, Basar, E, 2014. Decrease of event-related delta oscillations in euthymic patients with bipolar disorder. Psychiatry Res. 223 (1), 43-48.

Auer, D.P., Putz, B., Kraft, E., Lipinski, B., Schill, J., Holsboer, F., 2000. Reduced glutamate in the anterior cingulate cortex in depression: an in vivo proton magnetic resonance spectroscopy study. Biol. Psychiatry 47 (4), 305-313.

Bramon, E., Sham, P.C., 2001. The common genetic liability between schizophrenia and bipolar disorder: a review. Curr. Psychiatry Rep. 3 (4), 332-337.

Brugger, S., Davis, J.M., Leucht, S., Stone, J.M., 2011. Proton magnetic resonance spectroscopy and illness stage in schizophrenia - a systematic review and meta-analysis. Biol. Psychiatry 69 (5), 495-503.

Bustillo, J.R., Chen, H., Jones, T., Lemke, N., Abbott, C., Qualls, C., Canive, J., Gasparovic, C., 2014. Increased glutamine in patients undergoing long-term treatment for schizophrenia: a proton magnetic resonance spectroscopy study at 3 T. JAMA Psychiatry 71 (3), 265-272.

Clinton, S.M., Meador-Woodruff, J.H., 2004. Abnormalities of the NMDA receptor and associated intracellular molecules in the thalamus in schizophrenia and bipolar disorder. Neuropsychopharmacology 29 (7), 1353-1362.

Colla, M., Schubert, F., Bubner, M., Heidenreich, J.O., Bajbouj, M., Seifert, F., Luborzewski, A. Heuser, I., Kronenberg, G., 2009. Glutamate as a spectroscopic marker of hippocampal structural plasticity is elevated in long-term euthymic bipolar patients on chronic lithium therapy and correlates inversely with diurnal cortisol. Mol. Psychiatry 14 (7), 696-704 (647).
Cooper, J., Bloom, F., Roth, R., 2003. The Biochemical Basis of Neuropharmacology. Oxford University Press, New York.

Coyle, J.T., 2006. Glutamate and schizophrenia: beyond the dopamine hypothesis. Cell. Mol. Neurobiol. 26 (4-6), 365-384.

Craddock, N., Owen, M.J., 2005. The beginning of the end for the Kraepelinian dichotomy. Br. J. Psychiatry 186, 364-366.

Cuthbert, B.N., 2014. The RDoC framework: facilitating transition from ICD/DSM to dimensional approaches that integrate neuroscience and psychopathology. World Psychiatry 13 (1), 28-35.

Dager, S.R., Friedman, S.D., Parow, A., Demopulos, C., Stoll, A.L., Lyoo, I.K., Dunner, D.L. Renshaw, P.F., 2004. Brain metabolic alterations in medication-free patients with bipolar disorder. Arch. Gen. Psychiatry 61 (5), 450-458.

Dierks, T., Linden, D.E., Jandl, M., Formisano, E., Goebel, R., Lanfermann, H., Singer, W., 1999. Activation of Heschl's gyrus during auditory hallucinations. Neuron 22 (3), 615-621.

Domjan, N., Csifcsak, G., Drotos, G., Janka, Z., Szendi, I., 2012. Different patterns of auditory information processing deficits in chronic schizophrenia and bipolar disorder with psychotic features. Schizophr. Res. 139 (1-3), 253-259.

Ethridge, L.E., Hamm, J.P., Shapiro, J.R., Summerfelt, A.T., Keedy, S.K., Stevens, M.C., Pearlson, G., Tamminga, C.A., Boutros, N.N., Sweeney, J.A., Keshavan, M.S., Thaker, G., Clementz, B.A., 2012. Neural activations during auditory oddball processing discriminating schizophrenia and psychotic bipolar disorder. Biol. Psychiatry 72 (9), 766-774.

First, M.D., Gibbon, M., Spitzer, R.L., Gibbon, M., Williams, J.B.W., 1996. User's Guide for the Structured Interview for DSM-IV Axis I Disorders Research Version (SCID-I, version 2.0). Biometrics Research, New York.

Frey, B.N., Stanley, J.A., Nery, F.G., Monkul, E.S., Nicoletti, M.A., Chen, H.H., Hatch, J.P. Caetano, S.C., Ortiz, O., Kapczinski, F., Soares, J.C., 2007. Abnormal cellular energy and phospholipid metabolism in the left dorsolateral prefrontal cortex of medication-free individuals with bipolar disorder: an in vivo ${ }^{1} \mathrm{H}$ MRS study. Bipolar Disord. 9 (Suppl. 1), 119-127.

Gigante, A.D., Bond, D.J., Lafer, B., Lam, R.W., Young, L.T., Yatham, L.N., 2012. Brain glutamate levels measured by magnetic resonance spectroscopy in patients with bipolar disorder: a meta-analysis. Bipolar Disord. 14 (5), 478-487.

Goff, D.C., Coyle, J.T., 2001. The emerging role of glutamate in the pathophysiology and treatment of schizophrenia. Am. J. Psychiatry 158 (9), 1367-1377.

Hall, M.H., Schulze, K., Rijsdijk, F., Kalidindi, S., McDonald, C., Bramon, E., Murray, R.M., Sham, P., 2009. Are auditory P300 and duration MMN heritable and putative endophenotypes of psychotic bipolar disorder? A Maudsley Bipolar Twin and Family Study. Psychol. Med. 39 (8), 1277-1287.

Hamilton, M., 1960. A rating scale for depression. J. Neurol. Neurosurg. Psychiatry 23, 56-62.

Homan, P., Vermathen, P., Van Swam, C., Federspiel, A., Boesch, C., Strik, W., Dierks, T. Hubl, D., Kreis, R., 2014. Magnetic resonance spectroscopy investigations of functionally defined language areas in schizophrenia patients with and without auditory hallucinations. NeuroImage 94, 23-32.

Jardri, R., Pouchet, A., Pins, D., Thomas, P., 2011. Cortical activations during auditory verbal hallucinations in schizophrenia: a coordinate-based meta-analysis. Am. J. Psychiatry 168 (1), 73-81.

Javitt, D.C., 2009. When doors of perception close: bottom-up models of disrupted cognition in schizophrenia. Annu. Rev. Clin. Psychol. 5, 249-275.

Javitt, D.C., 2010. When doors of perception close: bottom-up models of disrupted cognition in schizophrenia. Annu. Rev. Clin. Psychol. 5, 249-275.

Javitt, D.C., Zukin, S.R., Heresco-Levy, U., Umbricht, D., 2012. Has an angel shown the way? Etiological and therapeutic implications of the PCP/NMDA model of schizophrenia. Schizophr. Bull. 38 (5), 958-966.

Kell, C.A., Morillon, B., Kouneiher, F., Giraud, A.L., 2011. Lateralization of speech production starts in sensory cortices - a possible sensory origin of cerebral left dominance for speech. Cereb. Cortex 21 (4), 932-937.

Kempton, M.J., Geddes, J.R., Ettinger, U., Williams, S.C., Grasby, P.M., 2008. Meta-analysis, database, and meta-regression of 98 structural imaging studies in bipolar disorder. Arch. Gen. Psychiatry 65 (9), 1017-1032.

Keshavan, M.S., Ongur, D., 2014. The journey from RDC/DSM diagnoses toward RDoC dimensions. World Psychiatry 13 (1), 44-46.

Kraguljac, N.V., Reid, M., White, D., Jones, R., den Hollander, J., Lowman, D., Lahti, A.C. 2012a. Neurometabolites in schizophrenia and bipolar disorder - a systematic review and meta-analysis. Psychiatry Res. 203 (2-3), 111-125.

Kraguljac, N.V., Reid, M.A., White, D.M., den Hollander, J., Lahti, A.C., 2012b. Regional decoupling of $\mathrm{N}$-acetyl-aspartate and glutamate in schizophrenia. Neuropsychopharmacology 37 (12), 2635-2642.

Kugaya, A., Sanacora, G., 2005. Beyond monoamines: glutamatergic function in mood disorders. CNS Spectr. 10 (10), 808-819.

Kuhn, S., Gallinat, J., 2012. Quantitative meta-analysis on state and trait aspects of auditory verbal hallucinations in schizophrenia. Schizophr. Bull. 38 (4), 779-786.

Machado-Vieira, R., Ibrahim, L., Henter, I.D., Zarate Jr., C.A., 2012. Novel glutamatergic agents for major depressive disorder and bipolar disorder. Pharmacol. Biochem. Behav. 100 (4), 678-687.

Marsman, A., van den Heuvel, M.P., Klomp, D.W., Kahn, R.S., Luijten, P.R., Hulshoff Pol, H.E. 2013. Glutamate in schizophrenia: a focused review and meta-analysis of (1)H-MRS studies. Schizophr. Bull. 39 (1), 120-129.

Modinos, G., Costafreda, S.G., van Tol, M.J., McGuire, P.K., Aleman, A., Allen, P., 2013. Neuroanatomy of auditory verbal hallucinations in schizophrenia: a quantitative metaanalysis of voxel-based morphometry studies. Cortex 49 (4), 1046-1055.

Moore, C.M., Frazier, J.A., Glod, C.A., Breeze, J.L., Dieterich, M., Finn, C.T., Frederick, B. Renshaw, P.F., 2007. Glutamine and glutamate levels in children and adolescents with bipolar disorder: a 4.0-T proton magnetic resonance spectroscopy study of the anterior cingulate cortex. J. Am. Acad. Child Adolesc. Psychiatry 46 (4), 524-534. 
Nenadic, I., Dietzek, M., Langbein, K., Rzanny, R., Gussew, A., Reichenbach, J.R., Sauer, H, Smesny, S., 2014. Superior temporal metabolic changes related to auditory hallucinations: a (31)P-MR spectroscopy study in antipsychotic-free schizophrenia patients. Brain Struct. Funct. 219 (5), 1869-1872.

Ohrmann, P., Siegmund, A., Suslow, T., Spitzberg, K., Kersting, A., Arolt, V., Heindel, W. Pfleiderer, B., 2005. Evidence for glutamatergic neuronal dysfunction in the prefrontal cortex in chronic but not in first-episode patients with schizophrenia: a proton magnetic resonance spectroscopy study. Schizophr. Res. 73 (2-3), 153-157.

Ohrmann, P., Siegmund, A., Suslow, T., Pedersen, A., Spitzberg, K., Kersting, A., Rothermundt, M., Arolt, V., Heindel, W., Pfleiderer, B., 2007. Cognitive impairment and in vivo metabolites in first-episode neuroleptic-naive and chronic medicated schizophrenic patients: a proton magnetic resonance spectroscopy study. J. Psychiatr. Res. 41 (8), 625-634.

Ohrmann, P., Kugel, H., Bauer, J., Siegmund, A., Kolkebeck, K., Suslow, T., Wiedl, K.H., Rothermundt, M., Arolt, V., Pedersen, A., 2008. Learning potential on the WCST in schizophrenia is related to the neuronal integrity of the anterior cingulate cortex as measured by proton magnetic resonance spectroscopy. Schizophr. Res. 106 (2-3), 156-163.

Oldfield, R.C., 1971. The assessment and analysis of handedness: the Edinburgh inventory. Neuropsychologia 9 (1), 97-113.

Ongur, D., Drevets, W.C., Price, J.L., 1998. Glial reduction in the subgenual prefrontal cortex in mood disorders. Proc. Natl. Acad. Sci. U. S. A. 95 (22), 13290-13295.

Ongur, D., Jensen, J.E., Prescot, A.P., Stork, C., Lundy, M., Cohen, B.M., Renshaw, P.F., 2008 Abnormal glutamatergic neurotransmission and neuronal-glial interactions in acute mania. Biol. Psychiatry 64 (8), 718-726.

Ongur, D., Prescot, A.P., Jensen, J.E., Cohen, B.M., Renshaw, P.F., 2009. Creatine abnormalities in schizophrenia and bipolar disorder. Psychiatry Res. 172 (1), 44-48.

Ongur, D., Haddad, S., Prescot, A.P., Jensen, J.E., Siburian, R., Cohen, B.M., Renshaw, P.F., Smoller, J.W., 2011. Relationship between genetic variation in the glutaminase gen GLS1 and brain glutamine/glutamate ratio measured in vivo. Biol. Psychiatry 70 (2) 169-174.

Oribe, N., Onitsuka, T., Hirano, S., Hirano, Y., Maekawa, T., Obayashi, C., Ueno, T., Kasai, K. Kanba, S., 2010. Differentiation between bipolar disorder and schizophrenia revealed by neural oscillation to speech sounds: an MEG study. Bipolar Disord. 12 (8), 804-812.

Overall, J.E., Gorham, D.R., 1962. The brief psychiatric rating scale. Psychological Reports 10, 799-812.

Paz, R.D., Tardito, S., Atzori, M., Tseng, K.Y., 2008. Glutamatergic dysfunction in schizophrenia: from basic neuroscience to clinical psychopharmacology. Eur. Neuropsychopharmacol. 18 (11), 773-786.

Plaze, M., Bartres-Faz, D., Martinot, J.L., Januel, D., Bellivier, F., De Beaurepaire, R., Chanraud, S., Andoh, J., Lefaucheur, J.P., Artiges, E., Pallier, C., Paillere-Martinot, M.L. 2006. Left superior temporal gyrus activation during sentence perception negatively correlates with auditory hallucination severity in schizophrenia patients. Schizophr. Res. 87 (1-3), 109-115.

Poels, E.M., Kegeles, L.S., Kantrowitz, J.T., Javitt, D.C., Lieberman, J.A., Abi-Dargham, A Girgis, R.R., 2014a. Glutamatergic abnormalities in schizophrenia: a review of proton MRS findings. Schizophr. Res. 152 (2-3), 325-332.

Poels, E.M., Kegeles, L.S., Kantrowitz, J.T., Slifstein, M., Javitt, D.C., Lieberman, J.A., AbiDargham, A., Girgis, R.R., 2014b. Imaging glutamate in schizophrenia: review of findings and implications for drug discovery. Mol. Psychiatry 19 (1), 20-29.

Provencher, S.W., 1993. Estimation of metabolite concentrations from localized in vivo proton NMR spectra. Magn. Reson. Med. 30 (6), 672-679.

Provencher, S.W., 2001. Automatic quantitation of localized in vivo ${ }^{1} \mathrm{H}$ spectra with LCModel. NMR Biomed. 14 (4), 260-264.

Rajarethinam, R.P., DeQuardo, J.R., Nalepa, R., Tandon, R., 2000. Superior temporal gyrus in schizophrenia: a volumetric magnetic resonance imaging study. Schizophr. Res. 41 (2), 303-312.

Rauschecker, J.P., Scott, S.K., 2009. Maps and streams in the auditory cortex: nonhuman primates illuminate human speech processing. Nat. Neurosci. 12 (6), 718-724.

Sanacora, G., Zarate, C.A., Krystal, J.H., Manji, H.K., 2008. Targeting the glutamatergic system to develop novel, improved therapeutics for mood disorders. Nat. Rev. Drug Discov. 7 (5), 426-437.

Schifitto, G, Zhong, J., Gill, D. Peterson, D.R., Gaugh, M.D., Zhu, T., Tivarus, M. Cruttenden, K., Maggirwar, S.B., Gendelman, H.E., Dewhurst, S., Gelbard, H.A., 2009. Lithium therapy for human immunodeficiency virus type 1-associated neurocognitive impairment. J. Neurovirol. 15 (2), 176-186.

Schwerk, A., Alves, F.D., Pouwels, P.J., van Amelsvoort, T., 2014. Metabolic alterations associated with schizophrenia: a critical evaluation of proton magnetic resonance spectroscopy studies. J. Neurochem. 128 (1), 1-87.
Seese, R.R., O'Neill, J., Hudkins, M., Siddarth, P., Levitt, J., Tseng, B., Wu, K.N., Caplan, R., 2011. Proton magnetic resonance spectroscopy and thought disorder in childhood schizophrenia. Schizophr. Res. 133 (1-3), 82-90.

Shenton, M.E., Kikinis, R., Jolesz, F.A., Pollak, S.D., LeMay, M., Wible, C.G., Hokama, H., Martin, J., Metcalf, D. Coleman, M., et al., 1992. Abnormalities of the left temporal lobe and thought disorder in schizophrenia. A quantitative magnetic resonance imaging study. N. Engl. J. Med. 327 (9), 604-612.

Shinn, A.K., Baker, J.T., Cohen, B.M., Ongur, D., 2013. Functional connectivity of left Heschl's gyrus in vulnerability to auditory hallucinations in schizophrenia. Schizophr. Res. 143 (2-3), 260-268.

Sikoglu, E.M., Jensen, J.E., Vitaliano, G., Liso Navarro, A.A., Renshaw, P.F., Frazier, J.A., Moore, C.M., 2013. Bioenergetic measurements in children with bipolar disorder: a pilot 31P magnetic resonance spectroscopy study. PLoS One 8 (1), e54536.

Silverstone, P.H., McGrath, B.M., 2009. Lithium and valproate and their possible effects on the myo-inositol second messenger system in healthy volunteers and bipolar patients. Int. Rev. Psychiatry 21 (4), 414-423.

Skold, M., Kallstrand, J., Nehlstedt, S., Nordin, A., Nielzen, S., Holmberg, J., Adolfsson, R., 2014. Thalamocortical abnormalities in auditory brainstem response patterns distinguish DSM-IV bipolar disorder type I from schizophrenia. J. Affect. Disord. 169, 105-111.

Skolnick, P., Popik, P., Trullas, R., 2009. Glutamate-based antidepressants: 20 years on. Trends Pharmacol. Sci. 30 (11), 563-569.

Soeiro-de-Souza, M.G., Salvadore, G., Moreno, R.A., Otaduy, M.C., Chaim, K.T., Gattaz, W.F., Zarate Jr., C.A., Machado-Vieira, R., 2013. Bcl-2 rs956572 polymorphism is associated with increased anterior cingulate cortical glutamate in euthymic bipolar I disorder. Neuropsychopharmacology 38 (3), 468-475.

Steen, R.G., Hamer, R.M., Lieberman, J.A., 2005. Measurement of brain metabolites by ${ }^{1} \mathrm{H}$ magnetic resonance spectroscopy in patients with schizophrenia: a systematic review and meta-analysis. Neuropsychopharmacology 30 (11), 1949-1962.

Szulc, A., Galinska, B., Tarasow, E., Waszkiewicz, N., Konarzewska, B., Poplawska, R., Bibulowicz, D., Simonienko, K., Walecki, J., 2011. Proton magnetic resonance spectroscopy study of brain metabolite changes after antipsychotic treatment. Pharmacopsychiatry 44 (4), 148-157.

Szulc, A., Galinska-Skok, B., Waszkiewicz, N., Bibulowicz, D., Konarzewska, B., Tarasow, E., 2013. Proton magnetic resonance spectroscopy changes after antipsychotic treatment. Curr. Med. Chem. 20 (3), 414-427.

Takahashi, T., Suzuki, M. Zhou, S.Y., Tanino, R. Hagino, H., Kawasaki, Y., Matsui, M., Seto, H., Kurachi, M., 2006. Morphologic alterations of the parcellated superior temporal gyrus in schizophrenia spectrum. Schizophr. Res. 83 (2-3), 131-143.

Tansey, F.A., Farooq, M., Cammer, W., 1991. Glutamine synthetase in oligodendrocytes and astrocytes: new biochemical and immunocytochemical evidence. J. Neurochem. 56 (1), 266-272.

Theberge, J., Jensen, J.E., Rowland, L.M., 2007. Regarding “Increased prefrontal and hippocampal glutamate concentration in schizophrenia: evidence from a magnetic resonance spectroscopy study". Biol. Psychiatry 61 (10), 1218-1219 (author reply 1219-1220).

Umbricht, D., Krljes, S., 2005. Mismatch negativity in schizophrenia: a meta-analysis. Schizophr. Res. 76 (1), 1-23.

Vita, A., De Peri, L., Deste, G., Sacchetti, E., 2012. Progressive loss of cortical gray matter in schizophrenia: a meta-analysis and meta-regression of longitudinal MRI studies. Transl. Psychiatry 2, e190.

Yamada, M., Wilber, J.F., 1990. Reciprocal regulation of preprothyrotropin-releasing hormone (TRH) mRNA in the rat anterior hypothalamus by thyroid hormone: dissociation from TRH concentrations during hypothyroidism. Neuropeptides 15 (1), 49-53.

Yatham, L.N., Kennedy, S.H., Schaffer, A., Parikh, S.V., Beaulieu, S., O'Donovan, C., MacOueen, G., McIntyre, R.S., Sharma, V., Ravindran, A., Young, L.T., Young, A.H., Alda, M., Milev, R., Vieta, E., Calabrese, J.R., Berk, M., Ha, K., Kapczinski, F., 2009. Canadian Network for Mood and Anxiety Treatments (CANMAT) and International Society for Bipolar Disorders (ISBD) collaborative update of CANMAT guidelines for the management of patients with bipolar disorder: update 2009. Bipolar Disord. 11 (3), 225-255.

Yildiz-Yesiloglu, A., Ankerst, D.P., 2006. Neurochemical alterations of the brain in bipolar disorder and their implications for pathophysiology: a systematic review of the in vivo proton magnetic resonance spectroscopy findings. Prog. Neuropsychopharmacol. Biol. Psychiatry 30 (6), 969-995

Young, R.C., Biggs, J.T., Ziegler, V.E., Meyer, D.A., 1978. A rating scale for mania: reliability, validity and sensitivity. Br. J. Psychiatry 133, 429-435.

Yuksel, C., Ongur, D., 2010. Magnetic resonance spectroscopy studies of glutamate-related abnormalities in mood disorders. Biol. Psychiatry 68 (9), 785-794.

Zatorre, R.J., Evans, A.C., Meyer, E., Gjedde, A., 1992. Lateralization of phonetic and pitch discrimination in speech processing. Science 256 (5058), 846-849. 